\title{
MELHORIA NO DESEMPENHO DO PROCESSO DE PRODUÇÃO DE PELOTAS DE MINÉRIO DE FERRO EM DISCOS DE PELOTIZAÇÃO PELA UTILIZAÇÃO DE SISTEMAS OTIMIZANTES COM LÓGICA NEBULOSA*
}

\author{
Luis Alberto Sfalsin Passos ${ }^{1}$ \\ João Luis Moreira² \\ Adilson Jorge ${ }^{3}$
}

Resumo

A aglomeração de minério de ferro para formação de pelotas é um processo dinâmico e seu controle é complexo. No controle manual o operador local é responsável pelos ajustes no processo e o tempo e a proporção de suas atuações dependem da percepção de cada um. Logo, o controle manual do processo é feito de forma individual e não padronizada e frequentemente não atende satisfatoriamente o controle da taxa de crescimento da pelota crua. Este artigo apresenta uma alternativa de controle automatizada, padronizada e otimizada da taxa de crescimento da pelota crua nos discos de pelotização. O "Sistema de Controle Avançado de Processo", denominado SCAP, consiste em um controle multivariável baseado em ferramentas de controle convencionais e lógica nebulosa, que simula o pensamento humano nas tomadas decisão. As regras foram construídas e fundamentadas em conhecimentos previamente adquiridos por especialistas do processo. Verificou-se após a implantação um aumento do desempenho do processo produtivo. No âmbito das melhorias, destacam-se: redução de pelotas fora da especificação (granulometria), redução do consumo de insumos (agentes aglomerantes, gás natural, entre outros), melhoria na qualidade do produto final e aumento da produtividade.

Palavras-chave: Aglomeração; Pelotas; Controle otimizante; Lógica nebulosa, Produtividade

\section{IMPROVING THE PERFORMANCE OF THE PRODUCTION PROCESS OF IRON ORE PELLETS IN DISCS PELLETIZING USING OPTIMIZING CONTROL SYSTEMS AND FUZZY LOGIC}

\begin{abstract}
The agglomeration of iron ore into pellets is a dynamic process and its control is complex. In manual control, the operator is responsible for adjusting lot of parameters, such as the control of the size ratio. All these parameters depend on the perception of each operator, based on its mindset. Thus, the manual control of the process is done individually and not standardized, and often this does not satisfactorily attend the green pellet growth rate. This article presents an alternative automated, standardized and optimized so as to control the green pellets growth rate in the pelletizing discs. The system, named "Advanced Process Control" or APC, consists of a multivariable control based on conventional control and fuzzy logic tools which simulates human thought in making decision. The rules were built and based on knowledge previously acquired by the experts of the process. It was verified, after the system implementation, a sharp increase in the process performance. The main improvements observed were: reduction of pellets out of specification (size distribution), reduction of raw materials consumption (binding agents, natural gas, etc.), improvement in the quality of the final product and productivity rise.

Keywords: Agglomeration; Pellets; Optimizing control; Fuzzy logic, Productivity.

1 Engenheiro de Produção, Engenheiro de Processo, Dep. Engenharia de Processo, Samarco Mineração S/A, Anchieta, ES, Brasil.

2 Engenheiro Metalúrgico e de Materiais, Laboratorista, Dep. Engenharia de Processo, Samarco Mineração S/A, Anchieta, ES, Brasil.

3 Engenheiro Elétrico, iTech Projetos Industriais LTDA, Belo Horizonte, MG, Brasil.

4 Engenheiro de Mecatrônica, Engenheiro de Processo, Dep. Engenharia de Processo, Samarco Mineração S/A, Anchieta, ES, Brasil.
\end{abstract}

\footnotetext{
* Contribuição técnica ao 44ำ Seminário de Redução de Minério de Ferro e Matérias-primas, 15ํ Simpósio Brasileiro de Minério de Ferro e $2^{\circ}$ Simpósio Brasileiro de Aglomeração de Minério de Ferro, 15 a 18 de setembro de 2014, Belo Horizonte, MG, Brasil.
} 


\section{INTRODUÇÃO}

As áreas de pelotamento da Samarco Mineração, por longo tempo, foram operadas de forma manual, onde as ações para controle do crescimento da pelota crua dependia unicamente da percepção dos operadores. Esse tipo de operação não é padrão para todos, pois depende da percepção que cada operador tem do processo naquele dado momento. Ocorre que os operadores estão em constante revezamento, seja pela troca de turno, troca de área ou novos entrantes. Estas constantes mudanças causam variação no processo produtivo. Além disto, novos operadores necessitam de um longo período de aprendizagem para tornarem-se capazes de operar a área, gerando perda de tempo e aumento nos custos operacionais.

Nos dias de hoje as empresas são, cada vez mais, obrigadas a crescer e aumentar a produtividade sem, contudo, comprometer a qualidade do produto final. Este fato exerce grande pressão sobre as operações manuais, fazendo com que busquem atualizar-se tecnologicamente e sejam capazes de produzir produtos dentro das especificações, com maior rapidez, menor variação e menor custo de produção.

Para atender a esta demanda, a Samarco Mineração investe no controle avançado para otimização dos seus processos produtivos. Os sistemas de controle avançado de processo estão entre os métodos mais eficientes de se otimizar os processos produtivos, minimizando os custos de produção, garantindo a produtividade e prazos. Os sistemas de controle avançado utilizam como entrada de dados os conhecimentos adquiridos, previamente, por pessoas especialistas das áreas operacionais e de engenharia. Além disto, para construção da estratégia de controle, os sistemas de controle avançado de processo se utilizam de ferramentas matemáticas avançadas, se comparados aos controles convencionais, tornando-os robustos e capazes de gerar incrementos com grande exatidão para controle.

Este artigo tem como objetivo apresentar a arquitetura do sistema otimizante, para controle da taxa de crescimento das pelotas cruas nos discos de pelototamento, e os ganhos provindos da sua utilização nas plantas de pelotização da Samarco Mineração SA. Sobretudo, mostra a capacidade de se reduzir a variabilidade dos processos, maximizando as pelotas cruas na granulometria entre $8,0 \mathrm{~mm}$ à $16,0 \mathrm{~mm}$, o que proporcionou um aumento na produtividade das plantas.

\section{MATERIAIS E MÉTODOS}

Segundo Meyer [1], o propósito do pelotamento é obter pelotas dentro da faixa de tamanho desejado e com resistência mecânica suficiente para serem transportadas seguramente dos discos de pelotamento para os equipamentos de queima. A etapa de aglomeração trata-se de um processo dinâmico, influenciado por características intrínsecas do minério e insumos, além de condições dependentes da atuação do operador para obtenção da faixa granulométrica desejada. A busca do controle de todas estas variáveis de processo é uma tarefa complexa. A figura 1 mostra como era a operação, manual, para controle da taxa de crescimento da pelota crua, antes da implantação do sistema de controle otimizante.

\footnotetext{
* Contribuição técnica ao 44 Seminário de Redução de Minério de Ferro e Matérias-primas, 15ㅇ Simpósio Brasileiro de Minério de Ferro e 2o Simpósio Brasileiro de Aglomeração de Minério de Ferro, 15 a 18 de setembro de 2014, Belo Horizonte, MG, Brasil.
} 
atendendo, tanto ao processo interno de endurecimento, quanto às qualidades físicoquímicas e metalúrgicas, exigidas pelos clientes, para a pelota queimada.

Fisicamente, para que as pelotas obtenham o tamanho e esfericidade dentro de uma especificação, elas devem percorrer um espaço dentro do disco.

Se esticarmos a trajetória que a pelota percorre dentro do disco em uma linha reta, veremos claramente que um fator primordial para seu crescimento é o espaço percorrido pela mesma.

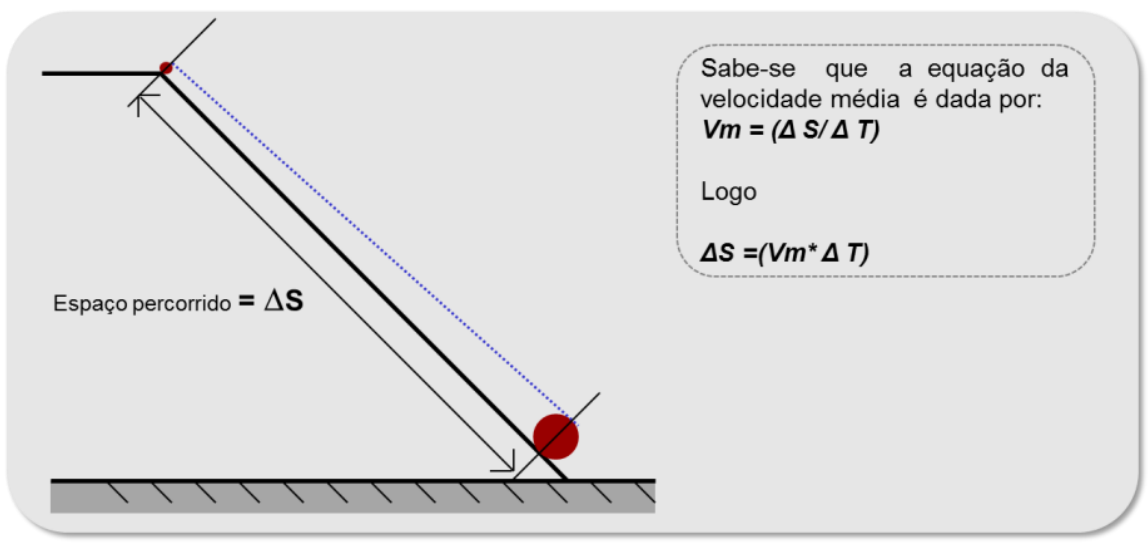

Figura 3 - Cinética de crescimento das pelotas verdes.

Então, para a partícula de minério percorrer um espaço " $\Delta S$ ", é preciso um intervalo de tempo " $\Delta T$ ", que é o tempo necessário para crescimento da pelota, como ilustrado na figura 3. Conclui-se que o espaço percorrido pelas pelotas nos discos é diretamente proporcional a velocidade e ao tempo de crescimento. Logo, quando se deseja aumentar o tamanho da pelota, aumenta-se a velocidade de rotação do disco e viceversa.

A composição da carga circulante dentro dos discos contém desde finos de minério à pelotas formadas no tamanho ideal. À medida que as pelotas crescem com 0 movimento de rotação dos discos, faz com que as pelotas menores fiquem no fundo e as maiores na superfície, sendo projetadas para fora do disco. A figura 4 exemplifica o comentado.

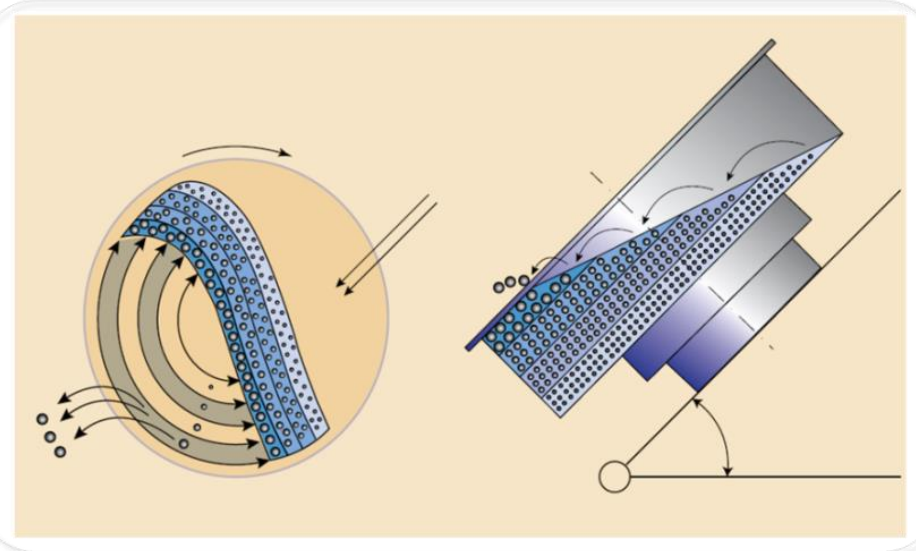

Figura 4 - Comportamento das pelotas dentro dos discos.

Observa-se que a alimentação dos discos é diretamente proporcional à produção desejada, e tem grande influência na taxa de crescimento das pelotas. À medida que a taxa de alimentação dos discos " $Q$ ” aumenta, em toneladas por hora, a velocidade

* Contribuição técnica ao 44 Seminário de Redução de Minério de Ferro e Matérias-primas, 15은 Simpósio Brasileiro de Minério de Ferro e $2^{\circ}$ Simpósio Brasileiro de Aglomeração de Minério de Ferro, 15 a 18 de setembro de 2014, Belo Horizonte, MG, Brasil. 
" $V$ " do fluxo de material dentro do disco aumenta, pois a área "A" da boca de saída do silo é constante. Se, " $Q=V^{*} A$ " então " $V=Q / A$ ". Logo, se a velocidade do fluxo é diretamente proporcional à taxa de alimentação, então, quando se aumenta a velocidade do fluxo, o tempo de permanência " $\Delta T$ " da pelota dentro do disco diminui, reduzindo o espaço percorrido " $\Delta S$ ", gerando pelotas com menores tamanhos. $\mathrm{Da}$ mesma forma, o inverso acontece quando se diminui a alimentação dos discos, cujas pelotas aumentam de tamanho. A figura 5 mostra os componentes do disco de pelotamento.

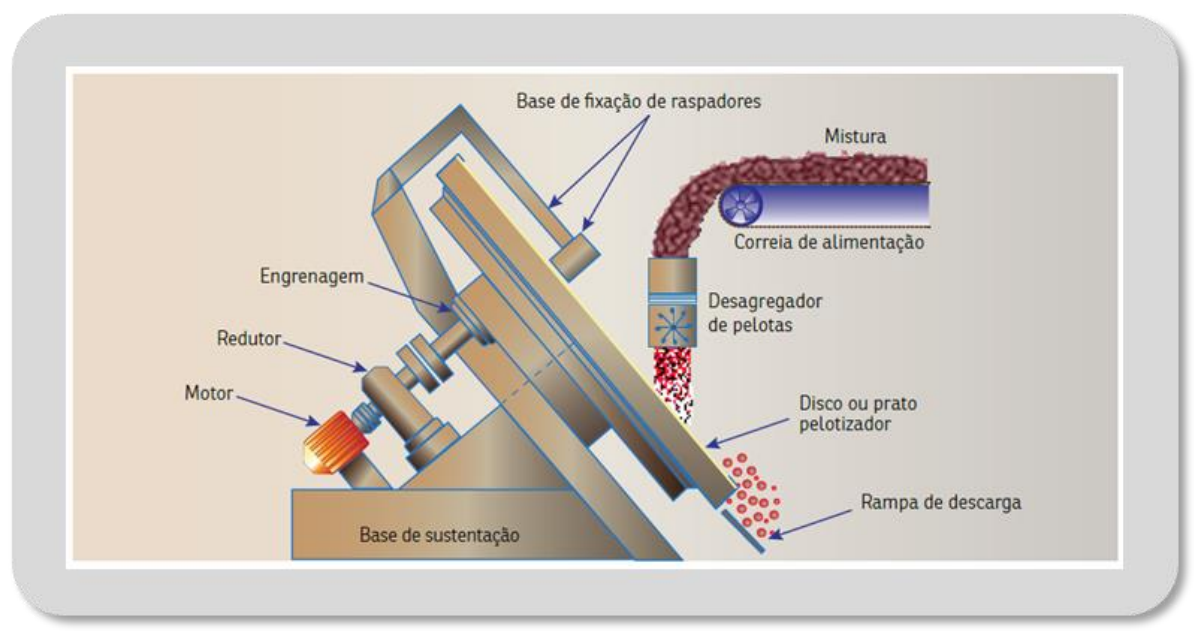

Figura 5 - Alimentação dos discos.

Importante frisar que o crescimento das pelotas também é influenciado pelas características do minério e insumos, como: umidade, granulometria, área superficial da partícula, agentes aglomerantes, etc.

\subsection{Arquitetura do Sistema}

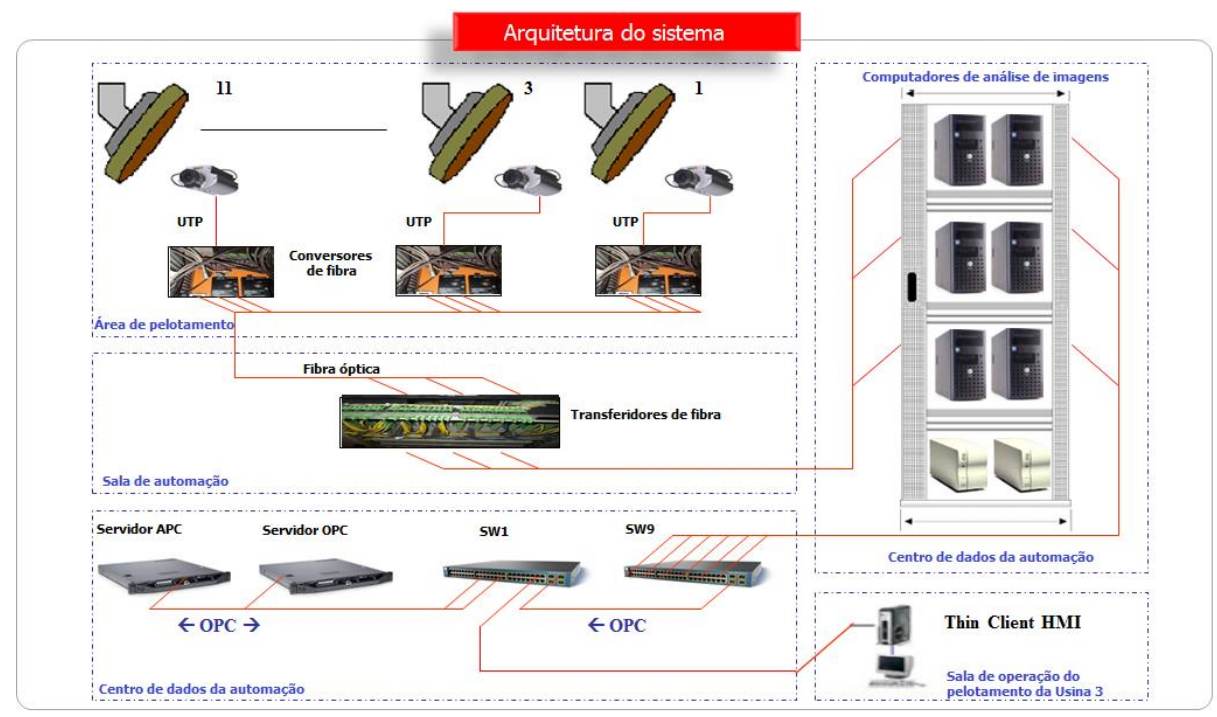

Figura 6 - Arquitetura do sistema de controle.

O sistema de controle avançado processo SCAP, utiliza com referência principal o monitoramento do tamanho das pelotas cruas via imagem. O sistema é formado por câmeras, redes de fibras óticas, conversores óticos, computadores analisadores de

\footnotetext{
* Contribuição técnica ao 44 Seminário de Redução de Minério de Ferro e Matérias-primas, 15ํ Simpósio Brasileiro de Minério de Ferro e $2^{\circ}$ Simpósio Brasileiro de Aglomeração de Minério de Ferro, 15 a 18 de setembro de 2014, Belo Horizonte, MG, Brasil.
} 
imagem, switches, servidor do SCAP, servidor de comunicação com o sistema de automação e interface de operação HMI (figura 6).

\subsection{Interface Homem Máquina HMI}

$\mathrm{Na}$ sala de controle do pelotamento encontra-se a interface homem maquina $\mathrm{HMI}$, que permite ao operador local realizar o ajuste da granulometria desejada para pelota crua, individualmente, em cada disco de pelotamento (figura 7).

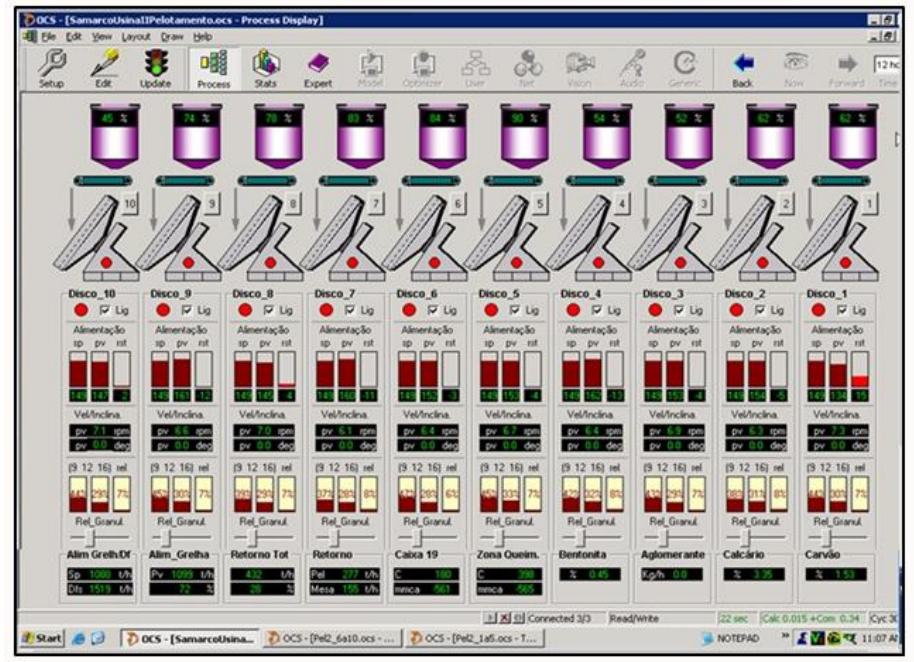

Figura 7 - Interface homem maquina "HMl".

\subsection{Captura e Tratamento de Imagem}

As pelotas cruas são descarregadas nas rampas dos discos de pelotamento, local onde as imagens são capturadas. Nas rampas dos discos existem conjuntos de câmeras, de alta velocidade, com iluminação a LED embutida. A qualidade da imagem é imprescindível para um bom controle da granulometria. Para isso, faz se necessário ter uma boa iluminação e uma equipe dedicada para manutenção e limpeza dos conjuntos de imagens. A atuação dos operadores é importante para manter as rampas dos discos sempre limpas. As câmeras dispõem de ajustes como: tempo de exposição, brilho, contraste, ganho, entre outros (figura 8).

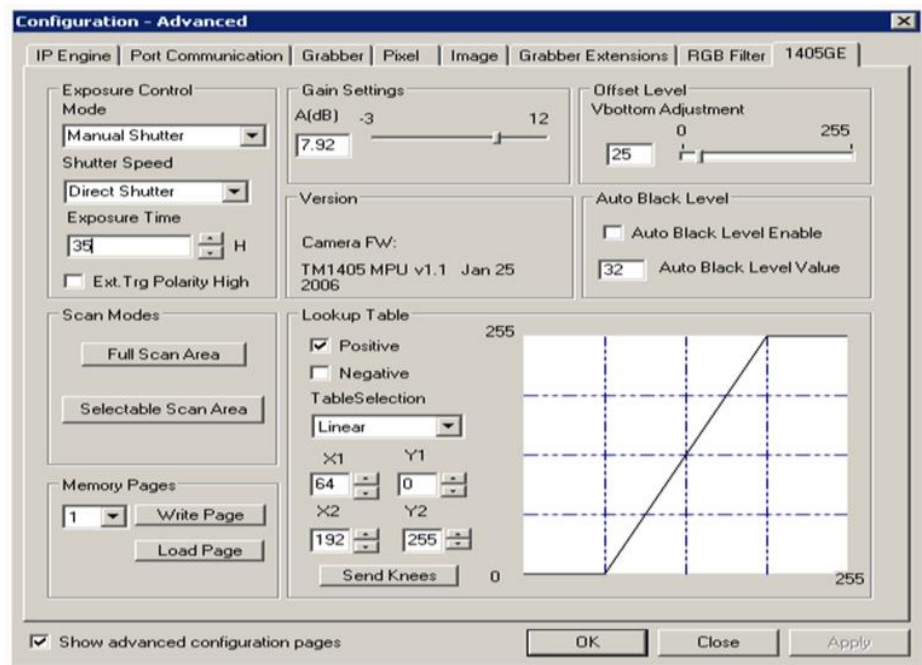

Figura 8 - Parâmetros de configuração do software Coyote (Camera - JAI PULNIX TM-1405GE).

\footnotetext{
* Contribuição técnica ao 44 Seminário de Redução de Minério de Ferro e Matérias-primas, 15ํ Simpósio Brasileiro de Minério de Ferro e $2^{\circ}$ Simpósio Brasileiro de Aglomeração de Minério de Ferro, 15 a 18 de setembro de 2014, Belo Horizonte, MG, Brasil.
} 
As imagens capturadas são enviadas aos computadores analisadores de imagens por meio de um link de fibra ótica ethernet TCP/IP. O SCAP comunica-se com computadores analisadores de imagem e sistema de automação de chão de fábrica, utilizando-se a tecnologia OPC (Ole for Process Control). As análises das imagens fornecem as referências de tamanho das pelotas cruas para o controle (figura 6).

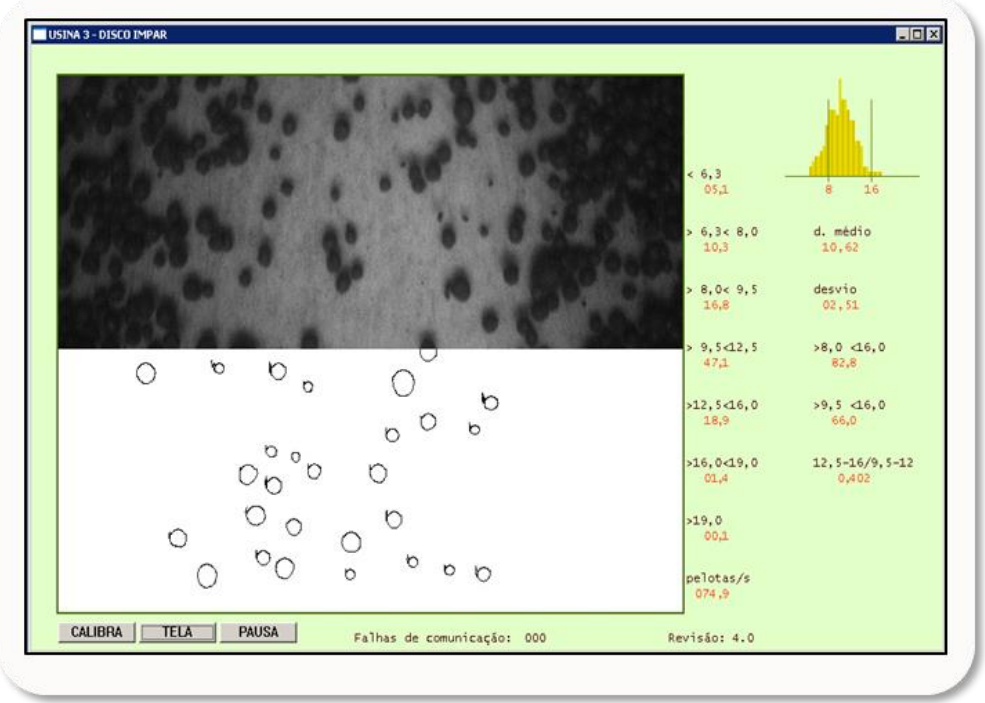

Figura 9 - Análise da distribuição granulométrica.

A análise das imagens é feita por um software que é responsável pela conversão da imagem em sete faixas granulométricas vide figura 9 . O processo de tratamento consiste em eliminação de ruídos (filtro passa baixa), detecção de bordas, algoritmo de amostragem (seleção), segregação das áreas fechadas em faixas granulométricas e geração da distribuição em percentual.

\subsection{Sistema de Controle Avançado SCAP}

As regras do SCAP foram elaboradas com base nos aprendizados adquiridos com os estudos de crescimento da pelota no disco de pelotização e os estudos para entendimento das práticas operacionais, manuais, para controle do pelotamento. As regras têm por objetivo controlar o tamanho da pelota atuando na velocidade e na alimentação dos discos.

\subsubsection{Controle da distribuição de carga entre os discos}

O SCAP também trabalha distribuindo carga entre os discos. A prioridade é direcionar mais carga para aqueles discos com melhor distribuição granulométrica e consequentemente melhor rendimento, vide figura 10. A distribuição de carga tem como meta o pedido geral de alimentação do pelotamento. É importante frisar que o rendimento do disco não é constante e depende das condições de desgaste de seus equipamentos, dos ajustes de parâmetros mecânicos e do material alimentado no mesmo. Logo, o rendimento do disco varia a todo tempo e a distribuição de carga funciona de forma dinâmica. O rendimento é dado pelo porcentual de pelotas produzidas na faixa entre $8 \mathrm{~mm}$ a $16 \mathrm{~mm}$.

\footnotetext{
* Contribuição técnica ao $44^{\circ}$ Seminário de Redução de Minério de Ferro e Matérias-primas, 15ํ Simpósio Brasileiro de Minério de Ferro e $2^{\circ}$ Simpósio Brasileiro de Aglomeração de Minério de Ferro, 15 a 18 de setembro de 2014, Belo Horizonte, MG, Brasil.
} 


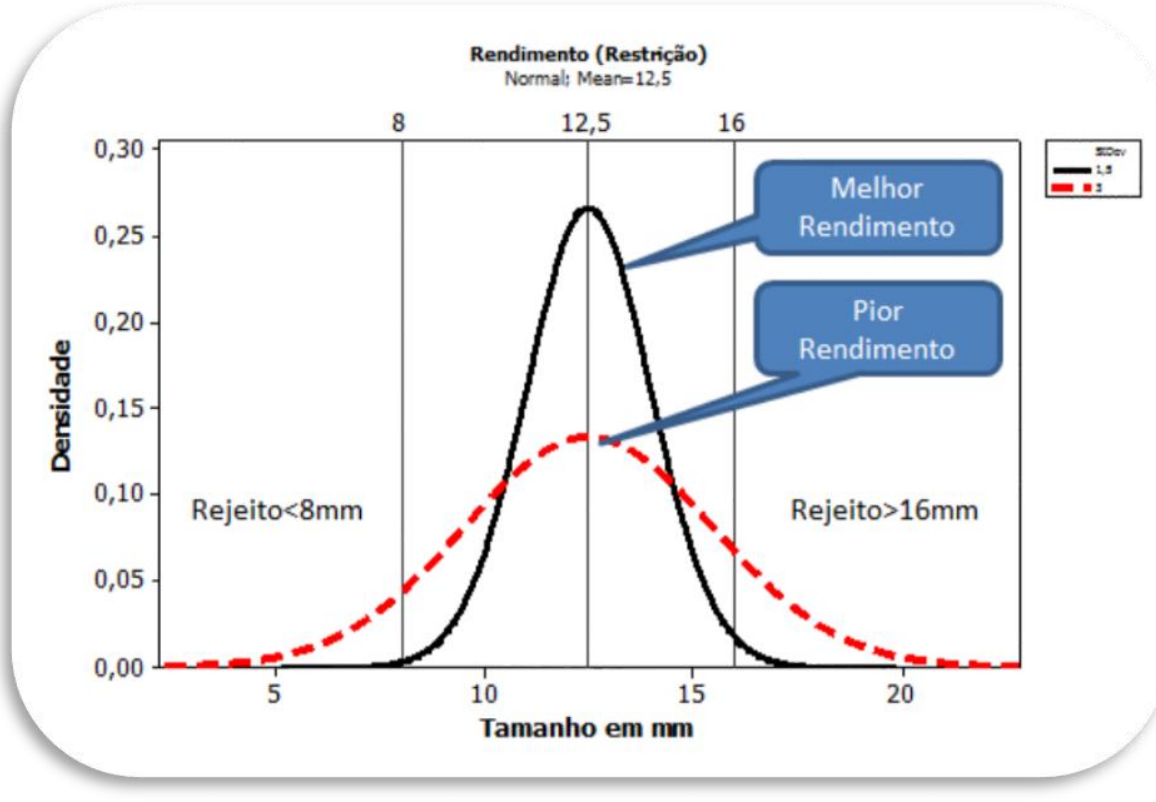

Figura 10 - Rendimento e distribuição de carga dos discos.

\subsubsection{Controle da relação granulométrica}

O SCAP processa as seguintes variáveis de entrada: o pedido de relação da pelota feito pelo operador, as informações de granulometria fornecidas pelos analisadores de imagem e as informações de velocidade e alimentação dos discos provenientes do sistema de automação. Após o processamento (cálculos e regras de controle), o SCAP devolve ao sistema de automação as variáveis de velocidade e alimentação dos discos, devidamente incrementadas. Por fim, cabe ao sistema de automação enviar as saídas que controlam os discos de pelotamento.

O SCAP processa as informações de entrada por meio de cálculos convencionais e cálculos estatísticos aliados a regras "crisp" e "fuzzy". Na estratégia de controle está contida a inteligência do sistema, que gera incrementos de saída com grande precisão de controle, vide figura 11.

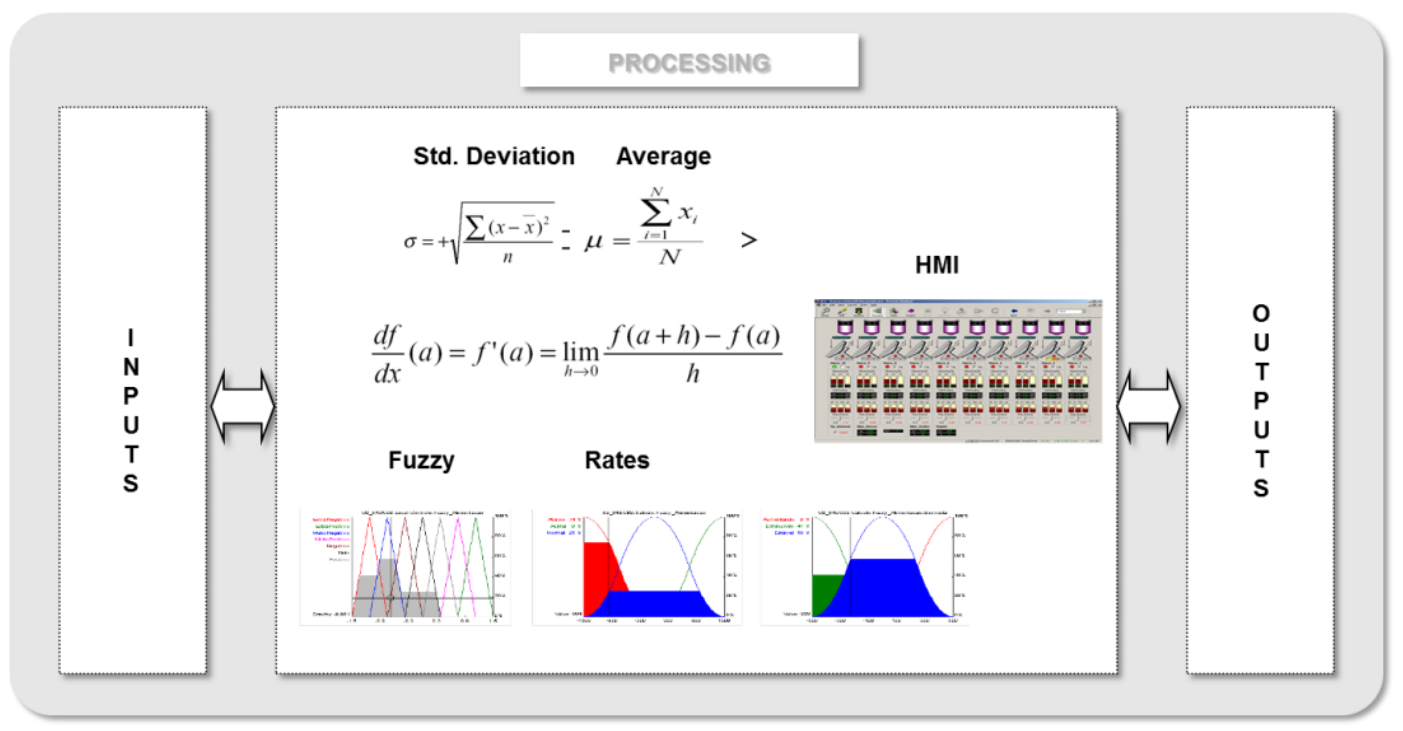

Figura 11 - Processamento do SCAP.

* Contribuição técnica ao $44^{\circ}$ Seminário de Redução de Minério de Ferro e Matérias-primas, 15ํ Simpósio Brasileiro de Minério de Ferro e $2^{\circ}$ Simpósio Brasileiro de Aglomeração de Minério de Ferro, 15 a 18 de setembro de 2014, Belo Horizonte, MG, Brasil. 


\section{RESULTADOS E DISCUSSÃO}

O SCAP é um sistema maduro e confiável na Samarco Mineração, mas para chegar a esse nível de maturidade foi necessária a criação uma equipe dedicada e multidisciplinar para Gestão do SCAP. A equipe é responsável por manter o sistema operante, com altos índices de disponibilidade e utilização.

\subsection{Ganho na Faixa de Pelotas de $8 \mathrm{~mm}$ a $16 \mathrm{~mm}$}

A figura 12 mostra uma redução na variabilidade e deslocamento da média, da faixa granulométrica de 8 a $16 \mathrm{~mm}$, para cima.
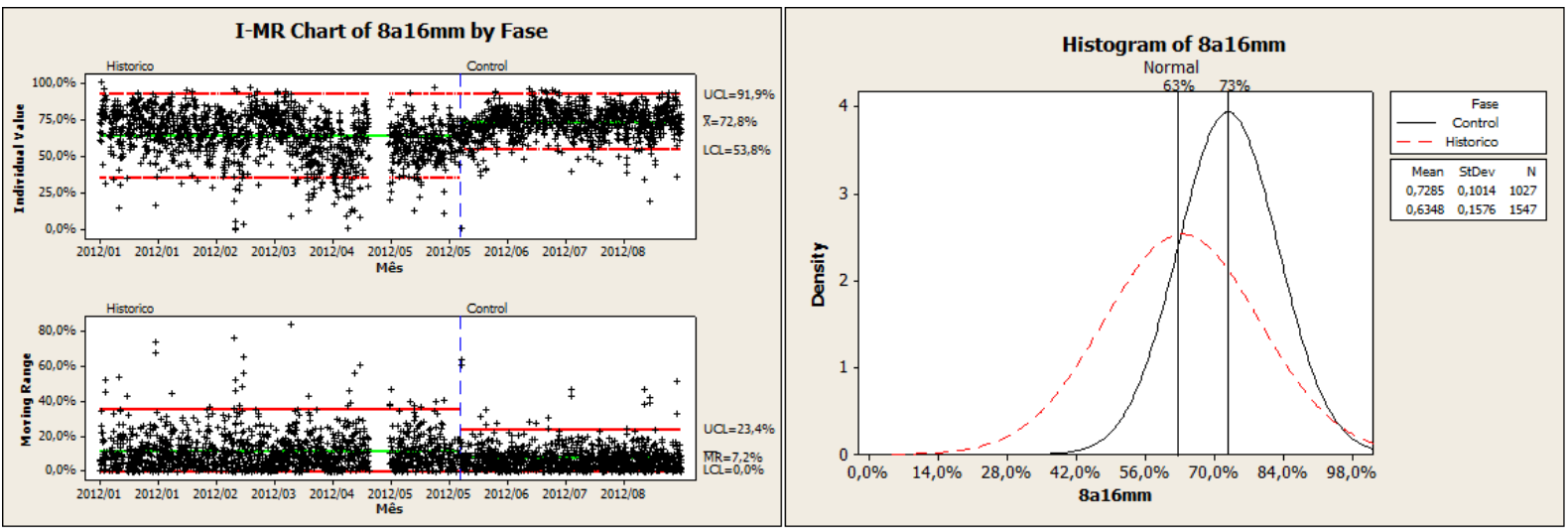

Figura 12 - Gráfico de redução da variabilidade e maximização das pelotas na faixa de $-16,0+8,0 \mathrm{~mm}$.

\subsection{Ganho de Estabilidade na Relação Granulométrica}

A figura 13 mostra uma redução na variabilidade da relação granulométrica.

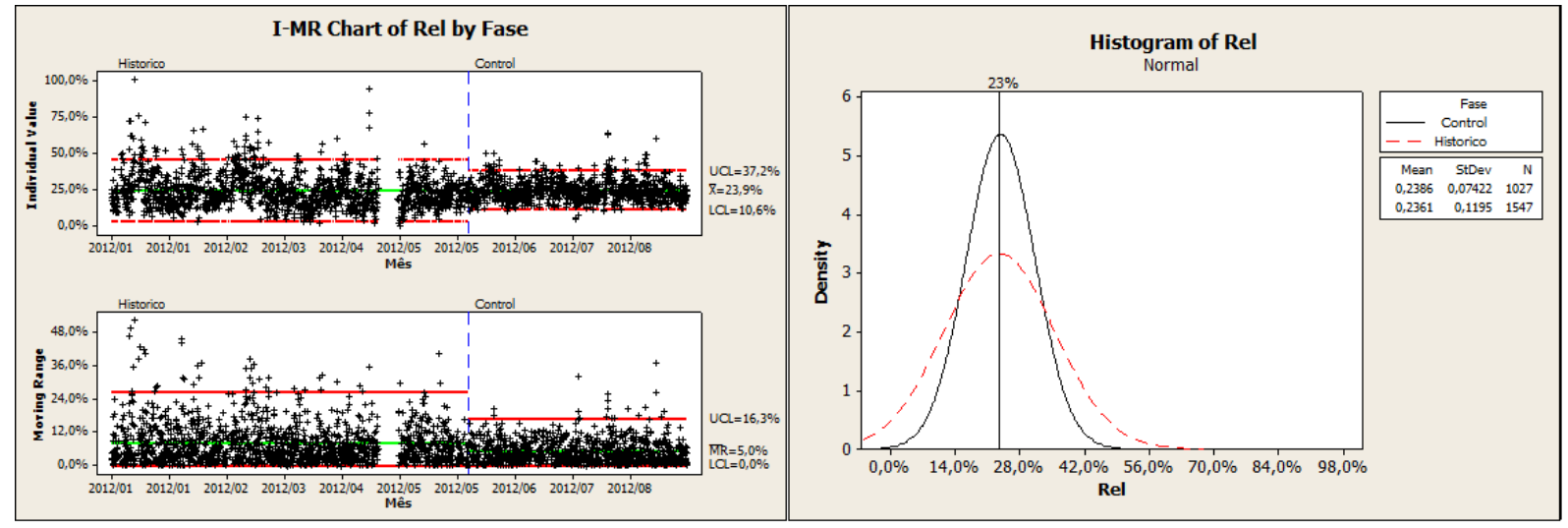

Figura 1 - Gráfico da redução da variabilidade da relação da Pelota Queimada.

A diferença da média entre os períodos é pequena e podemos considerar que não ocorreu mudança no valor médio do indicador. Na relação granulométrica não é interesse se deslocar a média, mas sim estreitar a distribuição.

\subsection{Ganho de Estabilidade no Diâmetro Média das Pelotas}

A figura 14 mostra uma redução na variabilidade do diâmetro média das pelotas.

* Contribuição técnica ao 44 Seminário de Redução de Minério de Ferro e Matérias-primas, 15오 Simpósio Brasileiro de Minério de Ferro e 2ํ Simpósio Brasileiro de Aglomeração de Minério de Ferro, 15 a 18 de setembro de 2014, Belo Horizonte, MG, Brasil. 

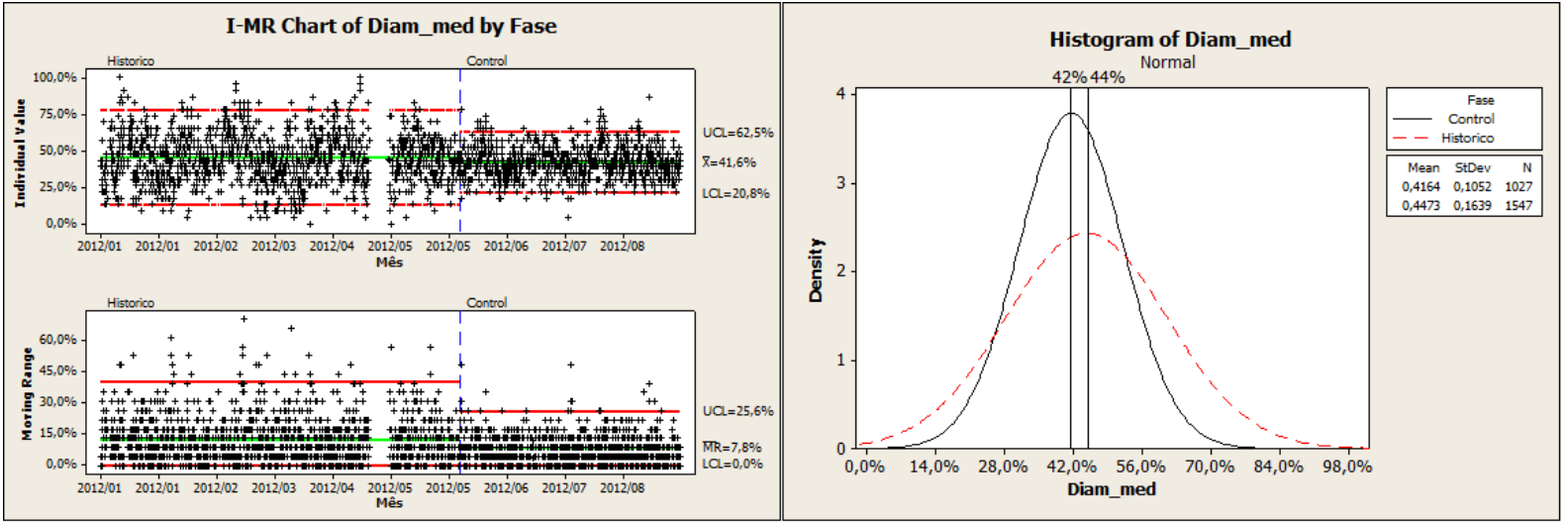

Figura 14 - Gráfico redução da variabilidade do diâmetro média das pelotas.

\subsection{Ganho no Consumo de Gás Natural}

A figura 15 mostra uma redução na variabilidade e deslocamento da média, do consumo de gás natural, para baixo.
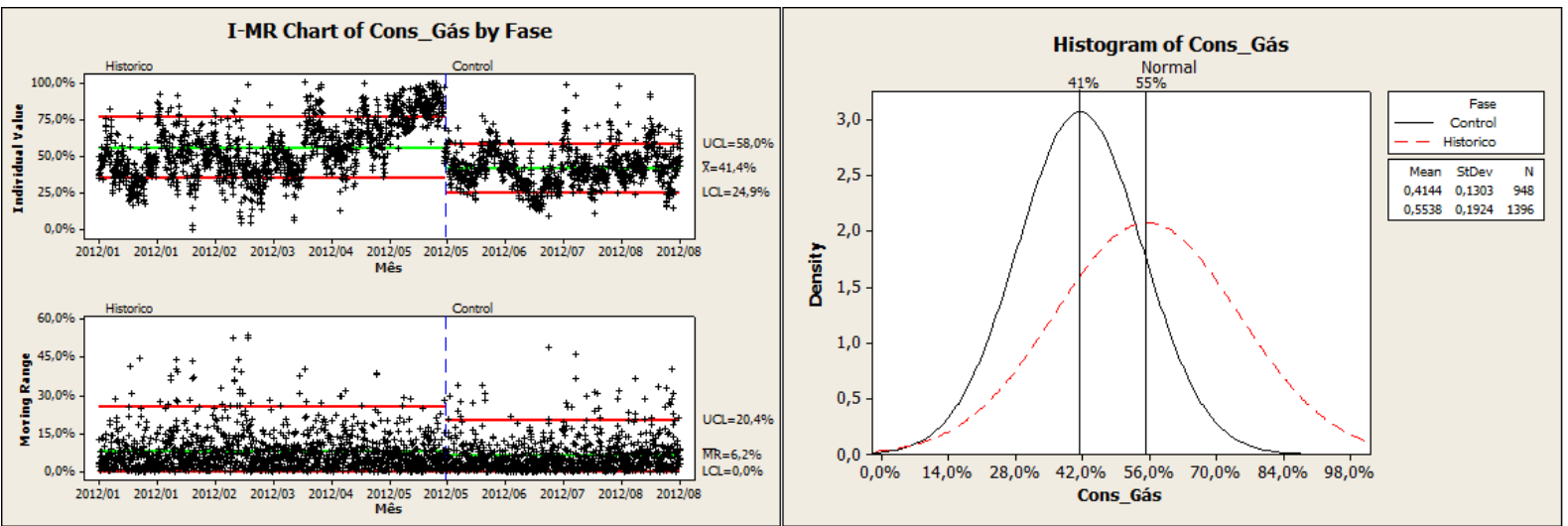

Figura 15 - Redução do consumo de gás.

\subsection{Ganho no Consumo do Insumo Bentonita}

A figura 16 mostra uma pequena redução na variabilidade e deslocamento da média, do consumo de bentonita, para baixo.
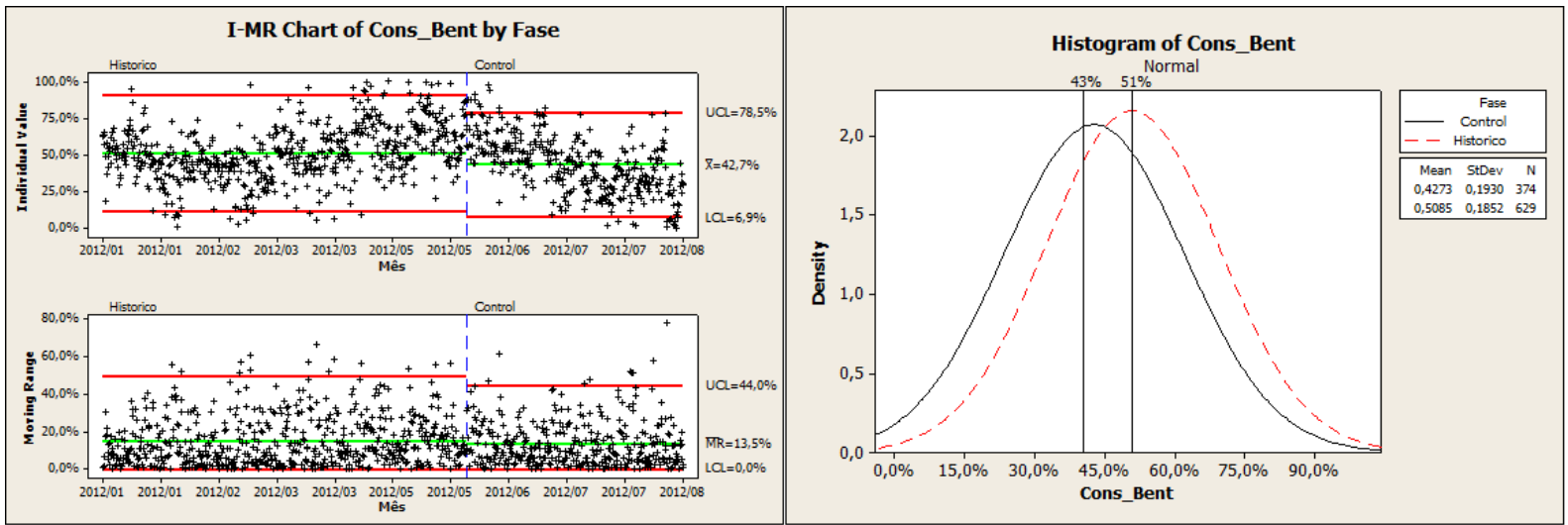

Figura 16 - Gráfico da redução do consumo de bentonita.

\subsection{Ganho no Consumo do Insumo Aglomerante Orgânico}

* Contribuição técnica ao $44^{\circ}$ Seminário de Redução de Minério de Ferro e Matérias-primas, 15은 Simpósio Brasileiro de Minério de Ferro e 20 Simpósio Brasileiro de Aglomeração de Minério de Ferro, 15 a 18 de setembro de 2014, Belo Horizonte, MG, Brasil. 
A figura 17 mostra uma pequena redução na variabilidade e deslocamento da média, do consumo de aglomerante orgânico, para baixo.
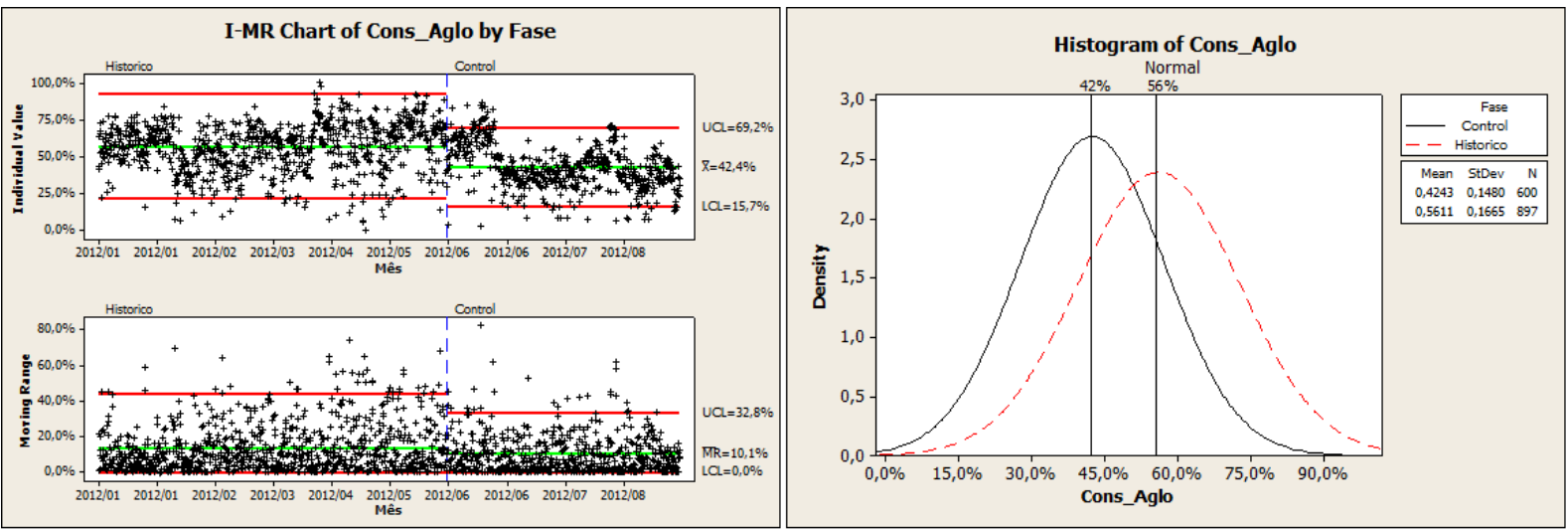

Figura 17 - Gráfico da redução do consumo de aglomerante orgânico.

\subsection{Ganho de Produção}

A figura 18 mostra uma redução na variabilidade e deslocamento da média, da alimentação da grelha do forno de pelotização, para cima.
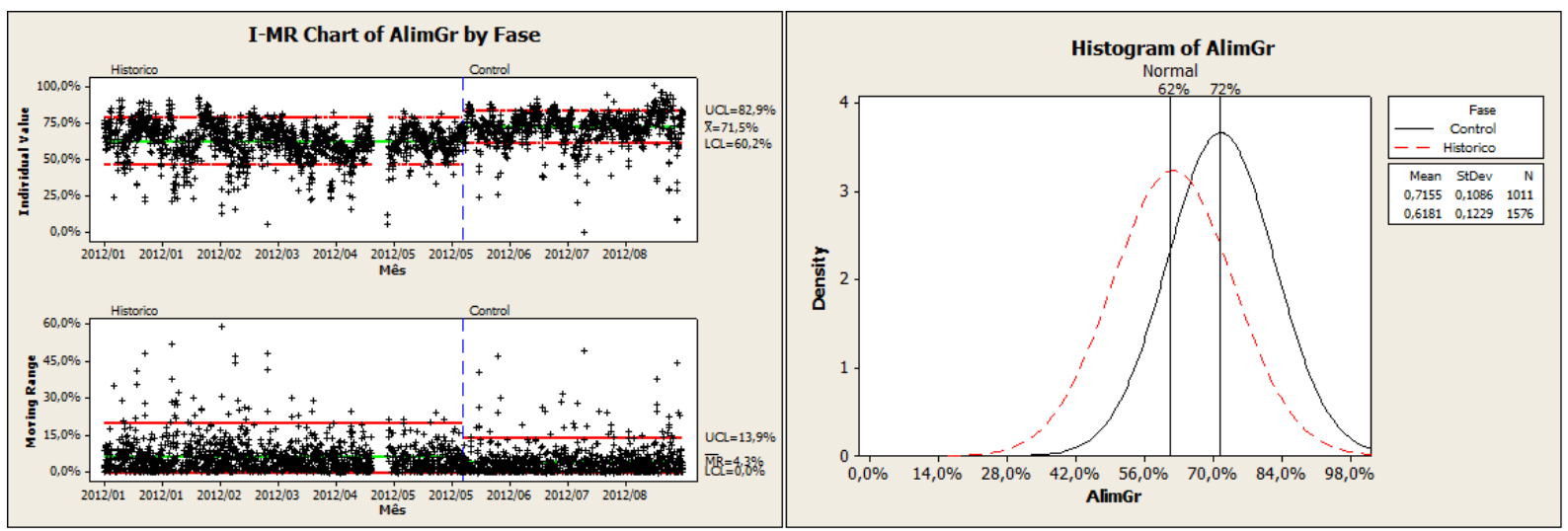

Figura 18 - Gráfico do aumento da alimentação da grelha de pelotas cruas.

\section{CONCLUSÃO}

O sistema de controle avançado de processo é uma alternativa de controle robusta e precisa. Os ganhos na redução da variabilidade proporcionados pelo SCAP, após sua implantação, são notórios se comparados aos dados históricos do processo. Destacam-se a relação granulométrica, a faixa granulométrica compreendida entre $8 \mathrm{~mm}$ a $16 \mathrm{~mm}$, e o diâmetro médio como motivadores dos demais ganhos. O controle destas variáveis proporcionou a estabilidade do processo produtivo, estabilidade da dosagem de insumos, aumento da permeabilidade do forno de endurecimento e consequentemente uma maior produtividade. Todos estes fatorem influenciam positivamente nos custos de produção. Porém, para obtenção dos ganhos reportados, é importante manter o sistema com alta disponibilidade e utilização. O SCAP foi aprovado para implantação em outras áreas da empresa.

\footnotetext{
* Contribuição técnica ao $44^{\circ}$ Seminário de Redução de Minério de Ferro e Matérias-primas, 15ํ Simpósio Brasileiro de Minério de Ferro e $2^{\circ}$ Simpósio Brasileiro de Aglomeração de Minério de Ferro, 15 a 18 de setembro de 2014, Belo Horizonte, MG, Brasil.
} 


\section{Agradecimentos}

Os autores agradecem a Samarco Mineração pelo suporte e incentivo

\section{REFERÊNCIAS}

1 Meyer K. Pelletizing of Iron Ores. Germany: Springer - Verlag Berlin Heidelberg New York, 1980.

\section{BIBLIOGRAFIA}

1 Sfalsin LAP. Seminário técnico para clientes da Samarco Mineração S/A, maio de 2014, Anchieta.

2 Gonçalves RG. Controle inteligente aplicado ao processo de queima de pelotas no forno PotGrate. Vitória: Universidade Federal do Espirito Santo, 2003.

3 Peroni DL. Estudo para reduzir a variabilidade do carbono fixo na alimentação de duas usinas de pelotização utilizando um controlador fuzzy. Vitória: Universidade Federal do Espirito Santo, 2006. 